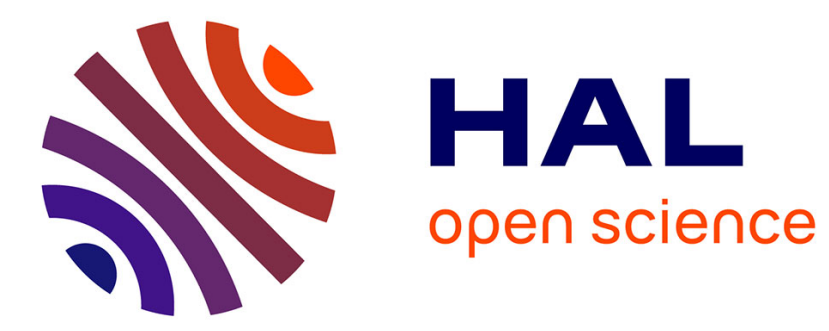

\title{
Spatial chaos of traveling waves has a given velocity
}

Bastien Fernandez, Beatriz Luna, Edgardo Ugalde

\section{To cite this version:}

Bastien Fernandez, Beatriz Luna, Edgardo Ugalde. Spatial chaos of traveling waves has a given velocity. Physical Review E: Statistical, Nonlinear, and Soft Matter Physics, 2009, 80, pp.025203(R). hal-00411530v2

\section{HAL Id: hal-00411530 \\ https://hal.science/hal-00411530v2}

Submitted on 12 Mar 2011

HAL is a multi-disciplinary open access archive for the deposit and dissemination of scientific research documents, whether they are published or not. The documents may come from teaching and research institutions in France or abroad, or from public or private research centers.
L'archive ouverte pluridisciplinaire HAL, est destinée au dépôt et à la diffusion de documents scientifiques de niveau recherche, publiés ou non, émanant des établissements d'enseignement et de recherche français ou étrangers, des laboratoires publics ou privés. 


\title{
Spatial chaos of traveling waves has a given velocity
}

\author{
Bastien Fernandez ${ }^{1}$, Beatriz Luna ${ }^{2}$ and Edgardo Ugalde $^{2}$ \\ 1 Centre de Physique Théorique*, CNRS Luminy Case 907, 13288 Marseille CEDEX 9, France \\ 2 Instituto de Física, Universidad Autónoma de San Luis Potosí, \\ Av. Manuel Nava 6, Zona Universitaria, 78290 San Luis Potosí, Mexico
}

\begin{abstract}
We study the complexity of stable waves in unidirectional bistable coupled map lattices as a test tube to spatial chaos of traveling patterns in open flows. Numerical calculations reveal that, grouping patterns into sets according to their velocity, at most one set of waves has positive topological entropy for fixed parameters. By using symbolic dynamics and shadowing, we analytically determine velocity-dependent parameter domains of existence of pattern families with positive entropy. These arguments provide a method to exhibit chaotic sets of stable waves with arbitrary velocity in extended systems.
\end{abstract}

PACS numbers: 05.45.Ra, 02.30.-f

Pattern formation in spatially extended systems can be regarded as the outcome of nonlinear dynamical processes [1]. This approach has proved successful to a vast phenomenology of various systems in Hydrodynamics, Optics, Solid-State Physics, etc. The most basic example concerns the spreading of simple coherent structures (e.g. fronts) in systems dominated by homogenization effects such as in reaction-diffusion [2] or in some examples of population dynamics [3]. In other circumstances, when localization effects are predominant, spatial chaos can emerge along with the production of cohorts of stable heterogeneous profiles, as in bistable optical devices [4] or in the Faraday instability of surface waves [5]. A breakthrough in the endeavor to elucidate this phenomenon has been to interpret solutions of stationary equations as the trajectories of a dynamical system where the original space variable plays the role of time. In this framework (which equally applies to continuous and discrete models) spatial chaos amounts to the existence of horseshoes and results from standard geometric constructions [6].

Beside standing patterns, extended systems also often generate a large variety of propagating structures especially when submitted to a global drift (e.g. open flows) [7]. In spite of this ubiquity, estimates on traveling pattern diversity are scarce, in particular as far as the velocity dependence is concerned. Indeed, stable waves with distinct velocities are known to possibly co-exist, but the occurrence of two or more chaotic sets of structures with distinct velocities remains unraveled. Appraising this issue from a theoretical viewpoint might have potential experimental applications in various fields, e.g. in fluid systems [7] or in secure communication [8]. In this context, the spatial dynamical system approach can also serve to construct horseshoes of traveling waves (TW) [9]. In principle, this tool also proves efficient to obtain

*UMR 6207 CNRS - Université Aix-Marseille II - Université AixMarseille I - Université Sud Toulon-Var conditions for chaos of waves with prescribed velocity (stationary solutions are regarded here as TW with vanishing velocity). However, since the velocity needs to be specified, it does not help to investigate the complexity dependence on velocity and parameters.

To gain intuition on these global features of spatial chaos in extended systems, we focus here on a minimal open flow model that can generate TW of arbitrary velocity, namely a unidirectional bistable Coupled Map Lattice (CML). Spatial chaos is quantified by the entropy of the sets of TW patterns with identical velocity. We numerically compute the entropy-velocity diagram in parameter space. The diagram unexpectedly reveals that spatial chaos occurs for at most a single velocity at once. To support this observation with analytical results, we rigorously infer, by means of symbolic dynamics, velocity-dependent parameter domains where specific TW pattern sets exist with positive entropy. These estimates show excellent agreement with numerical results and they extend to a larger class of models by structural stability. In a broader perspective, our shadowing arguments provide a new method to exhibit chaotic sets of stable waves with arbitrary velocity in extended systems.

The CML and its symbolic dynamics. We consider the unidirectional CML [7] on infinite real sequences,

$$
x_{s}^{t+1}=\left[F_{\epsilon}\left(x^{t}\right)\right]_{s}=(1-\epsilon) f\left(x_{s}^{t}\right)+\epsilon f\left(x_{s-1}^{t}\right)
$$

where $s \in \mathbb{Z}$ indexes lattice sites, $t$ represents time and the coupling constant $\epsilon$ lies in $[0,1]$. The individual map $f$ is piecewise linear, with two distinct branches separated by a jump at $x=T(0<T<1)$, i.e. $f(x)=a x+(1-a) H(x-T), H$ being the Heaviside function and $0 \leq a<1$. This type of bistable CML is reminiscent of gradient flows modeling advection phenomena in atmospheric circulation systems [10]. The dynamics under iterations of $f$ is elementary; any initial point located below the threshold $T$ monotonically converges to the fixed point 0 . Likewise, any point above $T$ is attracted by 1 .

The symbolic dynamics in this $\mathrm{CML}$ relies on the 
natural coding that consists in assigning a symbol 0 or 1 to each coordinate, according to its location with respect to $T$, namely let $\theta_{s}^{t}=H\left(x_{s}^{t}-T\right)$. In that way, symbolic codes can be explicitly inserted in (1) via $f\left(x_{s-r}^{t}\right)=a x_{s-r}^{t}+(1-a) \theta_{s-r}^{t}(r=0,1)$. By iterating backward the resulting expression, one gains a formula for the coordinates of orbits in the attractor (i.e. of those sequences $\left\{x^{t}\right\}$ whose components exist and are bounded for all $t \in \mathbb{Z}$ ) that exclusively depends on the code [11]

$$
x_{s}^{t}=\sum_{k=1}^{\infty} \sum_{n=0}^{k} \ell_{n, k} \theta_{s-n}^{t-k}
$$

where the coefficients $\ell_{n, k}=(1-a) a^{k-1}\left(\begin{array}{l}k \\ n\end{array}\right)(1-\epsilon)^{k-n} \epsilon^{n}$ are positive and normalized. The expression (2) is useful for two reasons. Firstly, it establishes a continuous bijection between codes and CML orbits; especially that given a symbolic sequence $\left\{\theta_{s}^{t}\right\}_{s, t \in \mathbb{Z}}$, there corresponds (if it exists) a single orbit $\left\{x_{s}^{t}\right\}_{s, t \in \mathbb{Z}}$ in the attractor. This correspondence commutes with $F_{\epsilon}$ and with space translations; hence all topological properties of the attractor express in symbolic codes. Secondly, (2) provides a substitute to the study of the attractor. An admissibility equation is obtained by requiring that the code computed based on (2) be indeed identical to the original symbolic sequence, i.e. a symbolic sequence $\left\{\theta_{s}^{t}\right\}$ codes for a genuine orbit iff the following relation holds

$$
H\left(\sum_{k=1}^{\infty} \sum_{n=0}^{k} \ell_{n, k} \theta_{s-n}^{t-k}-T\right)=\theta_{s}^{t}, \quad \forall s, t \in \mathbb{Z}
$$

Spatial complexity of traveling waves. Due to the advection term in (1), a characteristic feature of the CML is the prevalence of traveling patterns [7, 10]. Accordingly, in the analysis of eq. (3), we focus on symbolic sequences corresponding to traveling waves, i.e. $\theta_{s}^{t}=\theta_{\lfloor s-v t\rfloor}$ where $\lfloor\cdot\rfloor$ stands for the integer part [12]. This consideration substantially reduces the variety of solutions to study as these spatio-temporal sequences are parametrized by a purely spatial sequence $\left\{\theta_{s}\right\}$, called a pattern, and by a velocity $v$. However, TW seem to capture the essential characteristics of the attractor in our case. Of note, for $\epsilon=0$ any TW sequence with $v=0$ and arbitrary pattern in $\{0,1\}^{\mathbb{Z}}$ solves (3) (i.e. the uncoupled system $F_{0}$ has full diversity of fixed points), and similarly for $\epsilon=1$, any pattern is admissible with velocity $v=1$.

Another simplification comes from symmetries in parameter space. Beside the trivial exchange $\left(T, x_{s}^{t}\right) \leftrightarrow$ $\left(1-T, 1-x_{s}^{t}\right)$ (for orbits off discontinuities) which implies reflection invariance with respect to $T=1 / 2$, a more subtle symmetry exchanges any TW of velocity $v$ for $\epsilon$ with a TW of velocity $1-v$ for $1-\epsilon$ (reflection with respect to $\epsilon=1 / 2$ ). This property follows from the relation $F_{1-\epsilon} \circ R=\tau \circ R \circ F_{\epsilon}$ where $(R x)_{s}=x_{-s}$ and $(\tau x)_{s}=x_{s-1}$ are the spatial reflection and translation respectively.

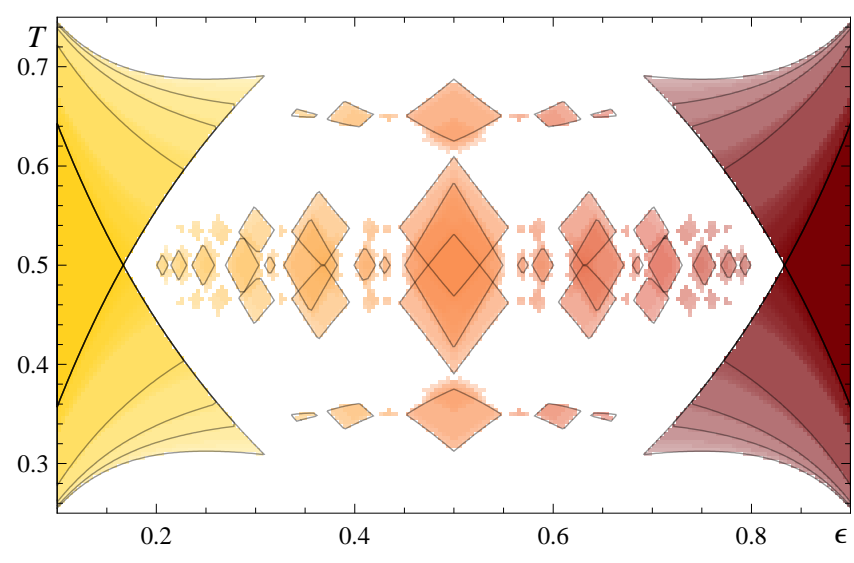

FIG. 1: Entropy-velocity diagram of stable TW in $(\epsilon, T)$-space for $a=0.8$. Painted points code for the numerical TW entropy $\frac{\log P_{19, v}}{19}$ (the period $L=19$ is chosen large enough for robustness). Gray levels (colors) correspond to velocities $v$, from 0 - left (light gray, yellow) to 1 - right (dark gray, brown) and including $\frac{1}{7}, \frac{1}{6}, \frac{1}{5}, \frac{1}{4}, \frac{2}{7}, \frac{1}{3}, \frac{2}{5}, \frac{3}{7}, \frac{1}{2}$ and symmetric values. Intensity is proportional to entropy (maximal values along the axis $T=0.5$ ). Curves delimit regions where TW symbolic sequences - with arbitrary pattern in a given chaotic set and given velocity - were analytically proved to solve the equation (3), see text. (Color online)

To get insights for arbitrary couplings, we have numerically estimated the $T W$ entropy [13] depending on the velocity (Fig. 1). The figure reveals a surprisingly elaborated structure where the entropy appears to be positive for at most a single velocity when all parameters are fixed, i.e. no overlapping colored points were found. Hence, chaos of stable TW has a given velocity in our system. This property is rather unexpected because some TW with distinct speeds are known to coexist here. The selected velocity increases from 0 to 1 with coupling $\epsilon$ as expected from the definition of $F_{\epsilon}$, and remains locked on rational values according to structural stability [14]. In particular, a velocity plot vs. $\epsilon$ for $T=1 / 2$ would show a Devil's staircase. Furthermore, the entropy itself remains constant on significant domains and surprisingly, besides reaching a maximum for symmetric maps $f$, this quantity for $0<v<1$ may also reach high values far away from $T=1 / 2$.

Fixed points analysis. To put numerical results on an analytic footing, we begin our investigations on eq. (3) by the spatial chaos of fixed points which materializes in the light gray (yellow) domain of Fig. 1. For a timeindependent code $\theta_{s}^{t}=\theta_{s}$, the orbit is obviously a fixed point whose coordinates are given by $x_{s}=\sum_{n=0}^{\infty} \ell_{n} \theta_{s-n}$ where

$\ell_{0}=\frac{(1-a)(1-\epsilon)}{1-a+a \epsilon} \quad$ and $\quad \ell_{n}=\frac{(1-a) \epsilon(a \epsilon)^{n-1}}{(1-a+a \epsilon)^{n+1}}, n>0$.

Eq. (3) stipulates that the coordinate $x_{s}$ at all sites $s$ where $\theta_{s}=1$ must exceed the threshold $T$ (for simplicity, we do not consider the case of coordinates lying on 
$T$ ). Likewise, all coordinates for which $\theta_{s}=0$ must lie below $T$. Since all coefficients $\ell_{n}$ are positive numbers, among all possible patterns and lattice sites, the smallest coordinate where $\theta_{s}=1$ is $x_{s}=\ell_{0}$. Using the normalization $\sum_{n=0}^{\infty} \ell_{n}=1$ and a similar argument for the sites where $\theta_{s}=0$, one concludes that when the inequalities $1-\ell_{0}<T<\ell_{0}$ hold, all patterns solve (3) with $v=0$. This condition is precisely the one that defines the central leftmost domain of maximal entropy $\log (2)$ in Fig. 1. In this case, no coordinate can cross the threshold $T$ under $F_{\epsilon}$, independently of the initial point in $[0,1]^{\mathbb{Z}}$, i.e. there cannot be any other admissible spatio-temporal sequence.

When $T$ is tuned out of the central leftmost domain, large sets of patterns disappear through analogues of saddle-node bifurcations [15] and the entropy decreases [16] as evidenced by changes in color intensity in Fig. 1. The entropy however does not gradually fall off to zero. It remains locked to positive values on plateaus and suffers abrupt changes on short scales. This staircase behavior can be analytically established for $a \leq 3 / 4$ (and under more general circumstances) as the fixed point bifurcation values belong to a Cantor set in this case. In addition, lower bounds on the entropy can be obtained by considering the sets, denoted by $A_{L}$ ( $L$ an arbitrary integer), that contain all patterns where any two consecutive 1 's are separated by at least $L$ consecutive 0 's. Starting from $A_{0}$ (which incorporates all patterns) these sets form a nested sequence with decreasing and asymptotically vanishing entropy as $L$ increases.

As for the admissibility of $A_{L}$, since there is no upper limitation on the 0-block length, the smallest coordinate for which $\theta_{s}=1$ in this set is again $x_{s}=\ell_{0}$. On the other hand, a straightforward argument based on positivity and decay of the coefficients $\ell_{n}$ shows that the largest coordinate for which $\theta_{s}=0$ is $x_{s}=m_{L} \equiv \sum_{n=0}^{\infty} \ell_{1+n(L+1)}$. Hence under the condition $m_{L}<T<\ell_{0}$ all patterns in $A_{L}$ are admissible with velocity 0 . The graphs of $m_{L}$ $(L=1,2,3)$ are shown in the leftmost domain in Fig. 1 and indicate that the sets $A_{L}$ indeed capture the main features of the fixed point entropy when $T$ decreases. Moreover, the fine structure of the fixed point entropy can be explained by considering more elaborated families with intermediate restrictions on block lengths (results not shown). Finally, the bottom curve delimiting the leftmost domain represents the graph of $m_{\infty}=\ell_{1}$, below which the entropy turns out to cancel (see below).

Intermediate velocities. Unlike for extreme velocities, when $v$ is fixed between 0 and 1 , no parameter value exists where the TW entropy is maximal. Nonetheless, it is possible to exhibit domains where the pattern entropy is positive by shadowing orbits with fronts and anti-fronts of the same velocity. In a preliminary step, proceeding similarly as for CML with diffusive couplings [11], one shows that a TW front code (defined as $\theta_{s}^{t}=H(s-v t), v$ rational) solves the admissibility equation iff the param- eters satisfy the inequalities $\eta(v-0)<T \leq \eta(v)$ where $\eta(v)=\sum_{k=1}^{\infty} \sum_{n=0}^{\lfloor v k\rfloor} \ell_{n, k}$ increases with $v$. By symmetry, a front coexists with its symmetric anti-front under the condition

$$
\omega(v) \equiv \max \{\eta(v-0), 1-\eta(v)\}<T<1-\omega(v) .
$$

This condition defines, for any rational velocity $v=\frac{p}{q}$ and any $0 \leq a<1$, a (symmetric with respect to $T=1 / 2)$ rhombus-like domain $S_{p / q}$ in the $(\epsilon, T)$-square. Moreover, these domains are pairwise disjoint and contiguous when $v$ changes as indicated in Fig. 1 .

To prove spatial chaos of TW, we consider the pattern set $B_{L}$ of positive entropy where all finite 0-blocks and all finite 1-blocks have length $L$ or larger. Any TW code with pattern in $B_{L}$ and velocity $\frac{p}{q}$ has the property to locally match, on any space-time window of size $L$, and modulo a time shift $t_{0}$, either the corresponding front code (i.e. we have $\theta_{s^{\prime}}^{t^{\prime}}=H\left(s^{\prime}-\frac{p}{q}\left(t^{\prime}-t_{0}\right)\right)$ for $s-L<s^{\prime} \leq s$ and $t-L<t^{\prime} \leq t$ ) or the anti-front code. According to (2) and to normalization of the $\ell_{n, k}$, the coordinate $x_{s}^{t}$ then turns out to be close to either the front coordinate, i.e.

$$
\left|x_{s}^{t}-\phi_{\text {front }}\left(s-\frac{p}{q}\left(t-t_{0}\right)\right)\right|<a^{L-1}
$$

or to the anti-front's coordinate. In addition, inside $S_{p / q}$, all front and anti-front coordinates are bounded away from the threshold $T$ uniformly in space-time, say at a distance $\delta>0$. By choosing $L$ large enough so that $a^{L-1}<\delta$ (i.e. $L \geq L_{0}$ ), the inequality (4) forces $x_{s}^{t}$ and $\phi_{\text {front }}\left(s-\frac{p}{q}\left(t-t_{0}\right)\right)$ to be either both above or both below the discontinuity $T$ (and similarly when $x_{s}^{t}$ is close to the anti-front coordinate), i.e. eq. (3) holds at arbitrary point $(s, t)$. We conclude that all patterns in $B_{L_{0}}$ are admissible with $v=\frac{p}{q}$ and a positive lower bound applies for the associated pattern entropy inside $S_{p / q}$.

A similar shadowing argument can be applied to patterns in the sets $A_{L}$ above to gain further domains with positive entropy. The reference orbit in this case is unique; it is the soliton coded by $\theta_{s}^{t}=\delta(s-v t)$ where $\delta$ stands for the Dirac distribution. In practice, we have determined soliton existence domains, analytically for the velocity $1 / 2$ and numerically for $v=1 / 3$ and $1 / 4$. Their cross-sections for $a=0.8$, called $D_{p / q}$, are represented on Fig. 1 (bottom rhombus-like domains). As before, an excellent agreement is found with the corresponding regions of numerically evidenced positive entropy. Finally, we note that the shadowing argument can be repeated with further orbits to get additional predictions on the entropy. For instance, the numerically computed existence domains for the two-site solitons with $v=1 / 2,1 / 3$ and $1 / 4$ are reported in Fig. 1 . These are the rhombuslike structures intersecting their respective symmetric domain $S_{p / q}$. 

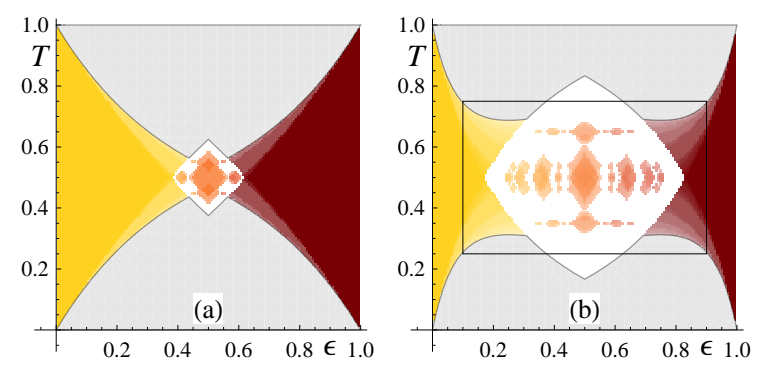

FIG. 2: Entropy-velocity diagram in the $(\epsilon, T)$-square and complementary domains where the entropy is analytically shown to cancel (outer light gray regions). (a) $a=0.4$ and (b) $a=0.8$; the rectangle delimits the region displayed in Fig. 1 . (Color online)

Completing the diagram. In addition to domains of positive entropy, we determined regions where the entropy cancels. In particular we proved that, under the condition $0<T<\min \left\{\ell_{0}, \ell_{1}\right\}$, (i) any symbol 1 remaining steady long enough at $s$, eventually forces the neighboring symbol at $s+1$ to become 1 and on the opposite (ii) the symbols 0 do not propagate (i.e. $\theta_{s}^{t}=1$ implies $\left.\theta_{s}^{t+1}=1\right)$. In this case, any code asymptotically approaches a sequence composed of front profiles (i.e. $\left.\theta_{s}^{t}=H(s-s(t))\right)$ and any TW entropy must vanish. By applying the symmetries $T \mapsto 1-T$ and $\epsilon \mapsto 1-\epsilon$, one obtains large domains of low spatial complexity in the parameter space, that share common boundaries with positive entropy domains as shown on Fig. 2. Of note, in the limit $a=0$ the CML becomes a cellular automaton. No TW with intermediate velocity exists in this case and there are four possible automaton rules according to the location of $(\epsilon, T)$ in the unit square.

Concluding remarks. Our analysis indicates that once the parameters are fixed in this system, various sets of TW co-exist with distinct velocities [17], but only a single ensemble can be chaotic. Numerical evidence has been supported by analytic arguments based on families of symbolic sequences. In a larger perspective, structural stability [14] implies that all chaotic sets exhibited here can be continued into suitable alterations of the piecewise affine model. Thus the existence of chaos of TW with arbitrary velocity generalizes to a larger class of bistable CML and beyond, to lattice systems with more general type of coupling (i.e. not fully asymmetric). Furthermore, based on uniqueness of the front velocity in bistable extended systems, we conjecture that the property of an at most one chaotic traveling patterns set holds in a broad context. Testing this property in further models provides a rewarding challenge for future studies.

Independently, our analytic methods proffer an alternative approach to the horseshoe construction, based on shadowing arguments. Indeed, the presence of a stable soliton (or the co-existence of stable fronts and anti-fronts of the same velocity) guarantees the existence of ensem- ble of traveling patterns with positive entropy. As it does not requires the explicit expression of localized solutions, this criterion is applicable to arbitrary spatially extended systems.

We are indebted to V. Afraimovich for constant stimulation and we thank C. Chandre, R. Coutinho and A. Torcini for a careful reading of the manuscript. We also acknowledge the host institutions for hospitality (i.e. BF to IF and BL and EU to CPT). Work supported by the ECOS-Nord programme M04M01.

[1] M.C. Cross and P.C. Hohenberg, Rev. Mod. Phys. 65 851 (1993).

[2] R. Kapral and R. Showalter, eds, Chemical Waves and Patterns, Kluwer (1995).

[3] J. Murray, Mathematical Biology. I: an introduction, Springer (2002).

[4] W.J. Firth, Phys. Rev. Lett. 61329 (1988).

[5] S. Douady, J. Fluid Mech. 221383 (1990).

[6] P. Coullet, C. Elphick and D. Repaux, Phys. Rev. Lett. 58431 (1987); J-P. Eckmann and I. Procaccia, Phys. Rev. Lett. 66891 (1991); V.I. Nekorkin and V.A. Makarov, Phys. Rev. Lett. 744819 (1995).

[7] F.H. Willeboordse and K. Kaneko, Phys. Rev. Lett. 73 533 (1994); O. Rudzick and A. Pikovsky, Phys. Rev. E 545107 (1996); G. Boffetta et.al., Phys. Rep. 356367 (2002).

[8] S. Wang et.al., Phys. Rev. E 66 065202(R) (2002).

[9] V.S. Afraimovich and V.I. Nekorkin, Int. J. Bif. Chaos 4 631 (1994).

[10] P.G. Lind, J. Corte-Real and J.A.C. Gallas, Phys. Rev. E 66016219 (2002); Int. J. Bif. Chaos 14999 (2004).

[11] R. Coutinho and B. Fernandez, Physica D 10660 (1997); Chaos 7301 (1997).

[12] By (2), the corresponding orbit coordinates write $x_{s}^{t}=$ $\phi(s-v t)$ where the profile is defined by $\phi(z)=$ $\sum_{k=1}^{\infty} \sum_{n=0}^{k} \ell_{n, k} \theta_{\lfloor z-n+v k\rfloor}$.

[13] For numerical purposes, the TW entropy is defined here as the exponential growth rate of the number $P_{L, v}$ of $L$-periodic patterns with a given velocity $v$, i.e. $\limsup _{L \rightarrow \infty} \frac{\log P_{L, v}}{L}$. This quantity is related to the standard topological entropy based on the number of admissible words, see e.g. R. Badii and A. Politi, Complexity. Hierarchical structures and scaling in physics, Cambridge University Press (1997).

[14] Because of contraction, any orbit remaining at bounded distance of discontinuities (as for all orbits considered here) is Lyapunov stable and thus structurally stable.

[15] B. Fernandez, J. Stat. Phys. 82931 (1996); M. Johnston, Phys. Lett. A 229156 (1997).

[16] For any $T<1-\ell_{0}=\sum_{n=1}^{\infty} \ell_{n}$, we have $T \leq \sum_{n=1}^{L} \ell_{n}$ for $L$ large enough. Thus, every fixed point pattern containing a (finite) 1-block of length $L$ or larger cannot be admissible and the fixed point entropy must be smaller than $\log (2)$.

[17] For instance fronts with velocity $v \neq 1 / 2$ co-exist together with a chaotic set of waves with velocity $1 / 2$ in the central bottom domain $D_{1 / 2}$ of Fig. 1 . 